\title{
RCTs inform on 5-year outcomes with TAVR and its use in patients at low surgical risk
}

O $\mathrm{n}$ the basis of the early results of the PARTNER randomized, controlled trials (RCTs) of transcatheter aortic valve replacement (TAVR) in patients with aortic stenosis considered to be at high risk or unsuitable for surgical aortic valve replacement (SAVR), TAVR is increasingly being used to treat patients with severe aortic stenosis. Findings from RCTs presented at the 2015 ACC Scientific Sessions, and simultaneously published online, have provided information about longer-term ( 5 year) outcomes in patients considered at high risk or unsuitable for SAVR, and about the use of this procedure in patients at lower surgical risk.

The prespecified final follow-up of patients enrolled in the PARTNER trials confirmed that the earlier findings of the study were maintained at 5 years. Among patients with severe aortic stenosis considered unsuitable for SAVR, TAVR was associated with a lower risk of death than standard treatment $(71.8 \%$ vs $93.6 \%$; HR 0.50, 95\% CI 0.39-0.65; $P<0.0001)$. Of the six patients in the standard treatment group who were alive at the 5-year follow-up, two had undergone TAVR, two had undergone SAVR, one had received an apical-descending aorta valve conduit, and one had undergone balloon aortic valvuloplasty. Median survival in the TAVR and standard treatment groups was 31.0 months and 11.7 months, respectively $(P<0.0001)$. The investigators confirmed that no continuous risk of stroke associated with TAVR existed after the initial procedure-related risk, and point out that the "valves were durable, with no increase in transvalvular gradient, attrition of valve area, or worsening of aortic regurgitation". In the PARTNER trial of patients considered at high surgical risk, the 5-year mortality was similar for the TAVR and SAVR groups (67.8\% vs 62.4\%; HR 1.04, 95\% CI $0.86-1.24 ; P=0.76)$. Median survival was 44.5 months and 40.6 months, respectively $(P=0.76)$. By 5 years, stroke rates were similar for the two treatment groups. Moderate-to-severe aortic regurgitation was previously shown to be more common with TAVR than with SAVR, mainly owing to paravalvular leak, and was associated with lower 5-year survival in the TAVR group. TAVR was associated with higher risk of major vascular complications, but lower risk of major bleeding complications, than SAVR; most of these complications occurred shortly after the transcatheter or surgical procedure. The investigators point out that the "TAVR was done with a firstgeneration device requiring a large sheathdelivery system in a very high surgical risk population; major advances in devices have since occurred". They say that "although not definitively proven, it is a reasonable expectation that newer generation devices might lead to better long term outcomes in terms of vascular complications and paravalvular leak." On the basis of their findings, the investigators conclude that TAVR should be considered an alternative to SAVR for the treatment of patients with aortic stenosis who are considered at high surgical risk.

The NOTION RCT was designed to determine the benefit and harms of TAVR in patients at lower surgical risk than previously assessed in an RCT setting. The investigators point out that "in recent years, there has been a trend to treat lowerrisk patients, and observational studies have demonstrated acceptable mortality outcomes in low- and intermediate-risk patients, but no RCTs have been conducted in this patient population.” NOTION was an all-comers trial of TAVR versus SAVR in 280 patients ( $\geq 70$ years) with echocardiographic severe, degenerative aortic valve stenosis. After the procedure and before discharge, the TAVR patients had lower rates of major or life-threatening bleeding, cardiogenic shock, and acute kidney injury, and were in hospital for a shorter period of time, than the individuals assigned to SAVR. "These differences ... reflect the less invasive nature of transcatheter treatment," note the investigators. Rates of major vascular complications did not significantly differ for the two groups. At both the 30-day and 1-year follow-ups, the rate of death, myocardial infarction, or stroke was similar for the two groups (at 1 year: 13.1\% for TAVR vs $16.3 \%$ for SAVR, $P=0.43$ for superiority; primary outcome measure), as were the rates of each of the separate components of the composite outcome measure. At the same time points, more patients who underwent TAVR had conduction abnormalities requiring a pacemaker, but fewer patients had newonset or worsening atrial fibrillation, compared with those who underwent SAVR. Dyspnoea was improved in both groups, but greater improvement was seen in the SAVR group than in the TAVR group. At 3 months and at 1 year, the TAVR patients had greater improvement in effective orifice area, but a higher rate of aortic valve regurgitation, than the SAVR patients. The NOTION investigators conclude that TAVR seemed safe and effective in low-risk and intermediaterisk patients. They state that "at present we are not able to recommend or refute one procedure over the other in lower risk patients", and caution that more randomized long-term data are needed before the indications for TAVR can be broadened to lower risk patients. Patients will be followed up for 5 years.

Bryony M. Mearns

Original articles Kapadia, S. R. et al. Lancet doi:10.1016/ S0140-6736(15)60290-2 | Mack, M. J. et al. Lancet doi:10.1016/S0140-6736(15)60308-7 | Thyregod, H. G. et al. J. Am. Coll. Cardiol. doi:10.1016/j.jacc.2015.03.014 